\title{
Controle de Anticarsia gemmatalis Hübner (Lepidoptera: Noctuidae) com reguladores de crescimento de insetos
}

\author{
Control of Anticarsia gemmatalis Hübner (Lepidoptera: Noctuidae) larvae with \\ insect growth regulators
}

Mauro Tadeu Braga da Silva ${ }^{1}$ Ervandil Corrêa Costa ${ }^{2}$ Adriano Boss $^{3}$

\section{RESUMO}

A lagarta-da-soja, Anticarsia gemmatalis Hübner (Lepidoptera: Noctuidae), é uma praga importante na cultura da soja no Brasil. Este estudo teve como objetivo avaliar diferentes inseticidas, reguladores de crescimento, para o controle dessa praga. Os inseticidas diflubenzuron (15g i.a. ha $\left.a^{-1}\right)$, lufenuron (7,5g i.a. $\left.h a^{-1}\right)$, metoxifenozide (21,6g i.a. $\left.h a^{-1}\right)$ e teflubenzuron (7,5g i.a. ha $\left.^{-1}\right)$ foram aplicados com pulverizador costal de precisão, equipado com pontas tipo leque 110, 02, numa pressão de 1 bar e volume de calda de $100 \mathrm{l} \mathrm{ha} \mathrm{h}^{-1}$. No dia da aplicação dos inseticidas, as plantas de soja (cv. Fundacep 33) estavam com 0,90m de altura, no estádio R3.1, e infestação média de 95 lagartas pequenas e de 33 lagartas grandes por dois metros de fileira. A eficiencia dos inseticidas foi determinada através da contagem do número de lagartas vivas pequenas e grandes aos 0, 2, 4, 7 e 10 dias após a aplicação (DAA); da percentagem de desfolha aos 15 DAA e da produção de grãos. Foi observado efeito de choque do inseticida metoxifenozide tanto em lagartas pequenas quanto em grandes. Os inseticidas diflubenzuron, lufenuron $e$ teflubenzuron controlaram eficientemente as lagartas a partir de $4 \mathrm{DAA}$. As infestações de lagartas nas parcelas testemunhas aumentaram a desfolha em 75,8\% e reduziram produtividade

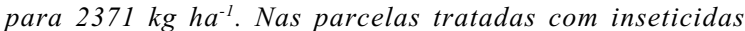
desfolha variou de $34,7 \%$ a $50,7 \%$ e a produtividade entre $3255 \mathrm{~kg} \mathrm{ha}^{-1}$ e $3585 \mathrm{~kg} \mathrm{ha}^{-1}$.

Palavras-chave: insecta, Glycine max L., inseticida, controle químico.

\section{ABSTRACT}

The velvetbean caterpillar, Anticarsia gemmatalis Hübner (Lepidoptera: Noctuidae), is an important soybean pest in Brazil. This study aimed to evaluate growth regulators insecticides. A precision sprayer equipped with 11002 nozzles, working at a constant pressure of 1 bar and volume of 100 liters per hectare was used to apply the insecticide treatments (diflubenzuron at $15 \mathrm{~g}$ a.i. per hectare, lufenuron at $7.5 \mathrm{~g}$ a.i. per hectare, metoxyfenozide at $21.6 \mathrm{~g}$ a.i. per hectare and teflubenzuron at $7.5 \mathrm{~g}$ a.i. per hectare). Applications when plants were $0.90 \mathrm{~m}$ height, in the $R 3.1$ growth stage, and the number of small larvae was 95, while that of large larvae was 33 per two meters of row. The number of live larvae at 0, 2, 4, 7 and 10 days after application, the defoliation at 15 days after application and grain yield were evaluated. The fastest control of large and small larvae was obtained with metoxyfenozide at 2 days after application. The insecticides diflubenzuron, lufenuron and teflubenzuron efficiently controlled of large and small larvae, but only at 4 days after application. Larval infestations in non-treated plots resulted in significant defoliation and yield losses when compared to plots treated with insecticides.

Key words: insecta, Glycine max L., insecticide, chemical control.

\section{INTRODUÇÃO}

A forma imatura de Anticarsia gemmatalis Hübner 1818 (Lepidoptera: Noctuidae) é conhecida por lagarta-da-soja. Dentre as lagartas desfolhadoras que ocorrem na cultura da soja no Brasil, essa espécie é a mais importante (PANIZZI \& CORRÊA-FERREIRA, 1997). Em plantas de soja, sob condições experimentais, as perdas, causadas pela praga, oscilaram entre 3 e

\footnotetext{
${ }^{1}$ Engenheiro Agrônomo, MSc, Pesquisador da FUNDACEP FECOTRIGO, CP 10, 98100-970, Cruz Alta-RS. E-mail: mtsilva.fundacep@comnet.com.br. Autor para correspondência.

${ }^{2}$ Engenheiro Agrônomo, Doutor, Pesquisador do CNPq, Professor Titular, Departamento de Defesa Fitossanitária, Centro de Ciências Rurais, Universidade Federal de Santa Maria, 97105-900, Santa Maria, RS. E-mail: eccosta@ccr.ufsm.br.

${ }^{3}$ Técnico Agrícola, Assistente de Pesquisa da FUNDACEP FECOTRIGO.
} 
$75 \%$, no período de oito safras agrícolas, nas parcelas não tratadas, considerando os métodos de controle biológico e químico e a associação de ambos (SILVA, 1992; 1993; 1995).

Diferentes ingredientes ativos foram pesquisados, nas condições brasileiras, para o controle químico dessa praga, destacando-se os inseticidas reguladores de crescimento de insetos, pela sua seletividade a inimigos naturais (SILVA et al., 1988). As benzoilfeniluréias (ex. diflubenzuron, flufenoxuron, lufenuron, nuvaluron, teflubenzuron $\mathrm{e}$ triflumuron) são os principais representantes dos inseticidas do grupo dos inibidores da síntese de quitina, Eles foram descobertos como venenos estomacais de ação lenta que interferem, especificamente, na deposição de quitina, um dos compostos da cutícula de insetos (REYNOLDS, 1987). Exercem sua ação tóxica em formas imaturas (larvas), particularmente durante a ecdise. RETNAKARAN et al. (1985) citam que estes compostos inibem a formação da quitina sintetase a partir de seu zimógeno, pela interferência em alguma protease responsável pela ativação da quitina sintetase. Assim, larvas tratadas com estes inseticidas não podem libertar-se de sua exocutícula, por não conseguirem secretar endocutícula nova. Recentemente, surgiram os inseticidas do grupo das diacilhidrazinas (ex. metoxifenozide e tebufenozide) que atuam como agonistas de ecdisteróides. Esses compostos provocam uma aceleração no processo da ecdise, porém, seu exato modo de ação ainda é desconhecido (DHADIALLA et al., 1998).

Considerando-se que os inseticidas reguladores de crescimento de insetos apresentam vantagens ecotoxicológicas sobre os inseticidas tradicionais, conduziu-se esta pesquisa com o objetivo de avaliar alguns desses inseticidas modernos, aplicados através da pulverização das plantas no controle da lagarta-da-soja.

\section{MATERIAL E MÉTODOS}

O experimento foi instalado no dia 03 de dezembro de 1999, em Cruz Alta - RS, com a semeadura do cultivar de soja Fundacep 33, feita com uma semeadora de parcela de quatro linhas, espaçadas em $0,40 \mathrm{~m}$, na densidade de 15 sementes por metro linear. $\mathrm{O}$ delineamento experimental foi o de blocos casualizados com 12 repetições, sendo as parcelas experimentais constituídas de 12 fileiras de soja por 10 $\mathrm{m}$ de comprimento. Os tratamentos constaram de diferentes inseticidas aplicados nas plantas (Tabelas 1, 2 e 3). Os inseticidas usados em pulverização das plantas foram aplicados com pulverizador costal de precisão, equipado com pontas leque 11002 Magnus ADGA, operando na pressão de 1 bar e volume de calda equivalente a $100 \mathrm{l} \mathrm{ha}^{-1}$. A pulverização dos inseticidas foi feita em 25 de fevereiro de 2000, com as plantas de soja no estádio R3.1 (FEHR et al., 1972), com altura de $0,90 \mathrm{~m}$ e desfolha de $20 \%$, em média, apresentando uma infestação aproximada de 95 e 33 lagartas pequenas e grandes, respectivamente, por dois metros de fileira.

Avaliou-se o número de lagartas vivas aos 0, 2, 4, 7 e 10 dias após a aplicação (DAA). Foram efetuadas três amostragens ao acaso por parcela, utilizando-se o pano de batidas (SHEPARD et al., 1974). O percentual de desfolha foi estimado através da observação visual de cada parcela aos 15 DAA. A produtividade de grãos foi determinada considerandose as seis fileiras centrais de cada parcela, eliminandose dois metros em cada extremidade e corrigindo-se a umidade em $13 \%$.

Os dados do número de lagartas amostradas foram transformados em raiz de $\mathrm{x}+0,5$ e os dados de percentagem de desfolha em arc sen raiz de $x \div 100$. Esses dados e os dados originais da produtividade de grãos foram submetidos à análise de variância e, posteriormente, ao teste de Tukey com significância em nível de $5 \%$ de probabilidade de erro. A percentagem de eficiência dos inseticidas foi calculada pela fórmula de HENDERSON \& TILTON (1955).

\section{RESULTADOS E DISCUSSÃO}

Pelas amostragens realizadas antes da aplicação dos inseticidas, a lagarta-da-soja estava presente de forma uniforme em toda área do experimento (Tabelas 1 e 2). Na testemunha, na qual não houve controle químico, o número de lagartas pequenas e grandes foi superior ao registrado nos tratamentos com inseticidas, em todas as amostragens efetuadas após a aplicação. Entre os inseticidas, as parcelas submetidas aos tratamentos com metoxifenozide apresentaram, consistentemente, menor número de lagartas pequenas e grandes, principalmente aos 2, 4, e 7 DAA, quando comparadas as parcelas tratadas com os inseticidas lufenuron, diflubenzuron e teflubenzuron.

A ocorrência contínua da lagarta-da-soja, durante todo período experimental, permitiu uma avaliação satisfatória dos tratamentos sobre a população da praga. As oviposições continuaram a ocorrer no experimento, como demonstrado pelo surgimento de lagartas pequenas na testemunha, principalmente aos 2 e 4 DAA (Tabela 1). Observou- 
Tabela 1 - Número $(\mathrm{N})$ de larvas pequenas $2 \mathrm{~m}^{-1}$ e percentagem de eficiência $(\% \mathrm{E})$ de inseticidas aplicados via pulverização de plantas no controle da lagarta-da-soja. Cruz Alta-RS, 2002.

\begin{tabular}{|c|c|c|c|c|c|c|c|c|c|}
\hline \multirow{3}{*}{ Tratamento (Dose) } & \multicolumn{9}{|c|}{ Dias após a aplicação (DAA) } \\
\hline & 0 & \multicolumn{2}{|c|}{2} & \multicolumn{2}{|c|}{4} & \multicolumn{2}{|c|}{7} & \multicolumn{2}{|c|}{10} \\
\hline & $\mathrm{N}$ & $\mathrm{N}$ & $\% \mathrm{E}$ & $\mathrm{N}$ & $\% \mathrm{E}$ & $\mathrm{N}$ & $\% \mathrm{E}$ & $\mathrm{N}$ & $\% \mathrm{E}$ \\
\hline Diflubenzuron ${ }^{1}(15)^{6}$ & $95,2^{7}$ & $97,0 a b^{8}$ & 18 & $28,8 \mathrm{~b}^{8}$ & 76 & $1,7 b c^{8}$ & 92 & $1,7 b^{8}$ & 86 \\
\hline Lufenuron $^{2}(7,5)^{6}$ & 94,7 & $77,2 \mathrm{~b}$ & 35 & $18,8 \mathrm{~b}$ & 84 & $1,8 \mathrm{bc}$ & 91 & $0,9 \mathrm{bc}$ & 93 \\
\hline Metoxifenozide $^{3}(21,6)^{6}$ & 94,7 & $17,2 \mathrm{c}$ & 85 & $0 \mathrm{c}$ & 100 & $0 \mathrm{c}$ & 100 & $0,1 \mathrm{c}$ & 99 \\
\hline Metoxifenozide $^{4}(21,6)^{6}$ & 96,7 & $19,7 \mathrm{c}$ & 84 & $0 \mathrm{c}$ & 100 & $0 \mathrm{c}$ & 100 & $0,1 \mathrm{c}$ & 99 \\
\hline Teflubenzuron ${ }^{5}(7,5)^{6}$ & 95,5 & $88,2 \mathrm{~b}$ & 26 & $30,0 \mathrm{~b}$ & 75 & $3,9 \mathrm{~b}$ & 81 & $1,9 \mathrm{~b}$ & 84 \\
\hline Testemunha & 96,1 & $118,7 \mathrm{a}$ & - & $118,4 \mathrm{a}$ & - & $21,0 \mathrm{a}$ & - & $12,2 \mathrm{a}$ & - \\
\hline $\mathrm{CV} \%$ & 11,3 & 12,3 & & 27,7 & & 45,1 & & $29,8 \mathrm{~b}$ & \\
\hline
\end{tabular}

${ }^{1}$ Dimilin PM (250g i.a. litro $\left.{ }^{-1}\right) ;{ }^{2}$ Match CE $\left(50\right.$ g i.a. quilo $\left.{ }^{-1}\right) ;{ }^{3}$ Valient SC $\left(240\right.$ g i.a. litro $\left.{ }^{-1}\right) ;{ }^{4}$ Intrepid SC $\left(240 \mathrm{~g}\right.$ i.a. litro $\left.{ }^{-1}\right) ;{ }^{5}$ Nomolt SC (150g i.a. litro $\left.^{-1}\right)$

${ }^{6}$ (g i.a. hectare $\left.\mathrm{e}^{-1}\right)$.

${ }^{7}$ Médias não significativas.

${ }^{8}$ Médias não seguidas da mesma letra diferem entre si pelo teste de Tukey em nível de 5\% de probabilidade de erro.

se uma redução contínua no número de lagartas pequenas nas parcelas tratadas, indicando que os inseticidas podem ter afetado a reprodução do inseto. ÁVILA \& NAKANO (1999) constataram efeito deletério de lufenuron sobre a capacidade de postura, fecundidade dos adultos e viabilidade de ovos de Diabrotica speciosa (Germar). Também ELEK \& LONGSTAFF (1994) não observaram geração F1 em Rhyzopertha dominica (L.), Sitophilus oryzae (L.), Oryzaephilus surinamensis (L.) e Tribolium castaneum (Herbst.), quando os adultos foram expostos a grãos de trigo tratados com os reguladores de crescimento chlorfluazuron, flufenoxuron, teflubenzuron e triflumuron. SCHROEDER \& SUTTON (1978) notaram menor viabilidade de ovos de Diaprepes abbreviatus (L.) após os adultos serem mantidos em contato com folhas de citros tratadas com diflubenzuron. BLUMBERG et al., (1985) evidenciaram efeito esterilizante de triflumuron sobre Carpophilus hemipterus (L.). Portanto, aumento nos percentuais de eficiência para lagartas pequenas (Tabela 1), no decorrer do experimento, aparentemente ocorreu pelo efeito dos inseticidas sobre a capacidade reprodutiva do inseto. Já o controle crescente de lagartas grandes (Tabela 2) até 10 DAA, pode ser

Tabela 2 - Número $(\mathrm{N})$ de larvas grandes $2 \mathrm{~m}^{-1}$ e percentagem de eficiência $(\% \mathrm{E})$ de inseticidas aplicados via pulverização de plantas no controle da lagarta-da-soja. Cruz Alta-RS, 2002.

\begin{tabular}{|c|c|c|c|c|c|c|c|c|c|}
\hline \multirow{3}{*}{ Tratamento (Dose) } & \multicolumn{9}{|c|}{ Dias após a aplicação (DAA) } \\
\hline & \multirow{2}{*}{$\begin{array}{c}0 \\
\mathrm{~N}\end{array}$} & \multicolumn{2}{|c|}{2} & \multicolumn{2}{|c|}{4} & \multicolumn{2}{|c|}{7} & \multicolumn{2}{|c|}{10} \\
\hline & & $\mathrm{N}$ & $\% \mathrm{E}$ & $\mathrm{N}$ & $\% \mathrm{E}$ & $\mathrm{N}$ & $\% \mathrm{E}$ & $\mathrm{N}$ & $\% \mathrm{E}$ \\
\hline Diflubenzuron $^{1}(15)^{6}$ & $35,9^{7}$ & $32,3 \mathrm{ab}^{8}$ & 24 & $8,2 \mathrm{c}^{8}$ & 81 & $3,7 b^{8}$ & 93 & $1,4 b^{8}$ & 86 \\
\hline Lufenuron $^{2}(7,5)^{6}$ & 35,4 & $26,8 \mathrm{~b}$ & 36 & $7,4 \mathrm{c}$ & 83 & $1,5 \mathrm{bc}$ & 97 & $0,6 \mathrm{bcd}$ & 94 \\
\hline Metoxifenozide $^{3}(21,6)^{6}$ & 33,7 & $5,4 \mathrm{c}$ & 87 & $0,2 \mathrm{~d}$ & 99 & $0 \mathrm{c}$ & 100 & $0 \mathrm{c}$ & 100 \\
\hline Metoxifenozide $^{4}(21,6)^{6}$ & 33,5 & $6,4 \mathrm{c}$ & 84 & $0,4 \mathrm{~d}$ & 99 & $0 \mathrm{c}$ & 100 & $0,2 \mathrm{~cd}$ & 98 \\
\hline Teflubenzuron ${ }^{5}(7,5)^{6}$ & 33,4 & $29,3 \mathrm{~b}$ & 26 & $13,5 \mathrm{~b}$ & 67 & $4,2 \mathrm{~b}$ & 91 & $1,1 \mathrm{bc}$ & 88 \\
\hline Testemunha & 33,8 & $40,1 \mathrm{a}$ & - & $41,0 \mathrm{a}$ & - & $48,3 \mathrm{a}$ & - & $9,5 \mathrm{a}$ & - \\
\hline $\mathrm{CV} \%$ & 10,9 & 14,7 & & 24,0 & & 35,4 & & $27,4 \mathrm{~b}$ & \\
\hline
\end{tabular}

${ }^{1}$ Dimilin PM (250g i.a. litro $\left.^{-1}\right) ;{ }^{2}$ Match CE $\left(50\right.$ g i.a. quilo $\left.{ }^{-1}\right) ;{ }^{3}$ Valient SC $\left(240\right.$ g i.a. litro $\left.^{-1}\right) ;{ }^{4}$ Intrepid SC $\left(240\right.$ g i.a. litro $\left.^{-1}\right) ;{ }^{5}$ Nomolt SC $\left(150\right.$ g i.a. litro $\left.^{-1}\right)$

${ }^{6}$ (g i.a. hectare $\left.{ }^{-1}\right)$.

${ }^{7}$ Médias não significativas.

${ }^{8}$ Médias não seguidas da mesma letra diferem entre si pelo teste de Tukey em nível de $5 \%$ de probabilidade de erro.

Ciência Rural, v. 33, n. 4, jul-ago, 2003. 
atribuído à maior mobilidade e/ou maior consumo alimentar à medida que essas se desenvolvem.

A ação da lagarta-da-soja causou maior percentual de desfolha na testemunha $(75,8 \%)$ quando comparada aos tratamentos com inseticidas. Os menores percentuais de desfolha $(34,7 \%$ e $34,8 \%)$ ocorreram nas parcelas aplicadas com metoxyfenozide e os maiores $(44,6 \%$ a $50,7 \%)$ nas parcelas com lufenuron, diflubenzuron e teflubenzuron. (Tabela 3). A menor produtividade de grãos de soja foi registrada na testemunha com $2371 \mathrm{~kg} \mathrm{ha}^{-1}$ quando comparadas com as parcelas tratadas (Tabela 3). Embora não houvesse diferença estatística entre os tratamentos com inseticidas, destacou-se o metoxyfenozide com produtividades maiores $\left(3569 \mathrm{~kg} \mathrm{ha}^{-1}\right.$ e $\left.3585 \mathrm{~kg} \mathrm{ha}^{-1}\right) \mathrm{em}$ relação ao lufenuron, diflubenzuron e teflubenzuron $\left(3255 \mathrm{~kg} \mathrm{ha}^{-1}\right.$ a $\left.3295 \mathrm{~kg} \mathrm{ha}^{-1}\right)$. Entre outros fatores, essa diferença aproximada de $9 \%$ na produtividade devese ao efeito de choque determinado pelos inseticidas à base de metoxyfenozide que, ao anteciparem a ecdise da lagarta-da-soja proporcionaram percentuais de controle significativamente superiores aos 2 DAA (Tabelas 1 e 2) e percentuais de desfolha significativamente inferiores às parcelas tratadas com inseticidas (Tabela 3).

As contagens de lagartas e as avaliações de desfolha e de produtividade indicam que todos os inseticidas reguladores de crescimento de insetos testados, nas doses utilizadas, permitem o controle adequado da lagarta-da-soja. Resultados semelhantes foram obtidos com diflubenzuron e teflubenzuron por HEINRICHS \& SILVA (1978), HEINRICHS et al.,(1979)

Tabela 3 - Percentagem de desfolha e produtividade de grãos de soja em diferentes tratamentos com e sem inseticidas usados para o controle da lagarta-da-soja. Cruz AltaRS, 2002.

\begin{tabular}{lccc}
\hline & Desfolha & \multicolumn{2}{c}{ Produtividade } \\
Tratamento (Dose) & $(\%)$ & $\mathrm{kg} \mathrm{ha}^{-1}$ & $\%$ Perda \\
\hline Diflubenzuron $^{1}(15)^{6}$ & $50,7 \mathrm{~b}^{7}$ & $3263 \mathrm{a}^{7}$ & 9,0 \\
Lufenuron $^{2}(7,5)^{6}$ & $44,6 \mathrm{~b}$ & $3255 \mathrm{a}$ & 9,2 \\
Metoxifenozide $^{3}(21,6)^{6}$ & $34,7 \mathrm{c}$ & $3569 \mathrm{a}$ & 0,5 \\
Metoxifenozide $^{4}(21,6)^{6}$ & $34,8 \mathrm{c}$ & $3585 \mathrm{a}$ & 0 \\
Teflubenzuron $^{5}(7,5)^{6}$ & $50,7 \mathrm{~b}$ & $3295 \mathrm{a}$ & 8,1 \\
Testemunha $_{\text {CV \% }}^{75,8 \mathrm{a}}$ & $2371 \mathrm{~b}$ & 33,9 \\
\hline
\end{tabular}

${ }^{1}$ Dimilin PM (250g i.a. litro $\left.{ }^{-1}\right){ }^{2}$ Match CE $\left(50\right.$ g i.a. quilo $\left.{ }^{-1}\right) ;{ }^{3}$ Valient SC (240g i.a. litro $\left.{ }^{-1}\right) ;{ }^{4}$ Intrepid SC $\left(240 \mathrm{~g}\right.$ i.a. litro $\left.{ }^{-1}\right) ;{ }^{5}$ Nomolt SC (150g i.a. litro $\left.{ }^{-1}\right)$.

${ }^{6}$ (g i.a. hectare $\left.{ }^{-1}\right)$.

${ }^{7}$ Médias não seguidas da mesma letra diferem entre si pelo teste de Tukey em nível de $5 \%$ de probabilidade de erro. e SILVA (1986). Estes resultados validam o uso de inseticidas reguladores de crescimento de insetos em substituição aos inseticidas convencionais, contribuindo para os programas de manejo integrado da lagarta-da-soja.

\section{CONCLUSÕES}

O regulador de crescimento de insetos metoxyfenozide possui efeito de choque sobre a lagarta-da-soja, enquanto diflubenzuron, lufenuron e teflubenzuron não confirmam esta ação.

Todos os inseticidas avaliados foram eficientes no controle da lagarta-da-soja, impedindo a ocorrência de dano econômico na cultura da soja.

\section{AGRADECIMENTOS}

Agradecemos ao pesquisador Giovani Theisen pelas sugestões em versões preliminares do manuscrito.

\section{REFERÊNCIAS BIBLIOGRÁFICAS}

ÁVILA, C.J.; NAKANO, O. Efeito do regulador de crescimento lufenuron na reprodução de Diabrotica speciosa (Germar) (Coleóptera: Chrysomelidae). Anais da Sociedade Entomológica do Brasil, Londrina, v.28, n.2, p.293-299, 1999.

BLUMBERG, D.; DORON, S.; BITTON, S. Effect of triflumuron on two species of nitidulit beetles, Carpophilus hemipterus and Urophorus humeralis. Phytoparasitica, v.13, p.9-19, 1985.

DHADiAlla, T.S.; CARLSON, G.R., LE, D.L. New insecticides with ecdysteroidal and juvenile hormone activity. Annual Review of Entomology, Palo Alto, v.43, p.545569,1998 .

ELEK, J.A.; LONGSTAFF, B.C. Effect of chitin-synthesis inhibitors on stored-products beetles. Pesticide Science, Chichester, v.40, p.225-230, 1994.

FEHR, W.R. et al. Stages of development descriptions for soybeans, Glycyne max (L.) Merril. Crop Science, Madison, v.11, p.929-931, 1972 .

HEINRICHS, E.A.; SILVA, R.F.P. da. Controle de Anticarsia gemmatalis Hübner, 1818 (Lepidoptera: Noctuidae) com $\mathrm{PH}$ 6040 em baixas dosagens. Agronomia Sulriograndense, Porto Alegre, v.14, n.2, p.261-267, 1978.

HEINRICHS, E.A.; GASTAL, H.A. de O.; GALILEO, M.H.M. Incidence of natural control agents of the velvetbean caterpillar and response of its predators to insecticide treatments in brazilian soybean fields. Pesquisa Agropecuária Brasileira, Brasília, v.14, n.1, p.79-87, 1979.

HENDERSON, C.F.; TILTON, E.W. Tests with acaricides against the brown wheat mite. Journal of Economic Entomology, Lanham, v.48, n.1, p.157-161, 1955. 
PANIZZI, A.R.; CORRÊA-FERREIRA, B. Dynamics in the insect fauna adaptation to soybean in the tropics. Trends in Entomology, v.1, p.71-88, 1997.

REYNOLDS, S.E. The cuticule, growth regulators and moulting in insects: the essential background to the action of acylurea insecticides. Pesticide Science, Chichester, v.20, p.131-146, 1987.

RETNAKARAN, A.; GRANETT, J.; ENNIS, T. Insect growth regulators. In: KERKUT, G.A.; GILBERT, L.I. Comprehensive insect physiology biochemistry and pharmacology. New York : Pergamon, 1985. Cap.12, p.529601

SCHROEDER, W.J.; SUTTON. R.A. Diaprepes abbreviatus: suppression of reproductive potential on citrus with an insect regulator plus spray oil. Journal of Economic Entomology, Lanham, v.71, p.69-70, 1978.

SHEPARD, M.; CARNER, G.R.; TURNIPSEED, S.G. A comparasion of three sampling methods for arthropds in soybeans. Environmental Entomology, Lanham, v.3, n.2, p.227-232, 1974
SILVA, M.T.B. da. Avaliação da eficiência de inseticidas biológicos e fisiológicos no controle da lagarta da soja. Revista Centro Ciência Rural, Santa Maria, v.16, n.2, p.151-160, 1986 .

SILVA, M.T.B. da. Associação de Baculovirus anticarsia com subdosagens de inseticidas no controle de lagartas de Anticarsia gemmatalis (Hübner, 1818). Ciência Rural, Santa Maria, v.25, n.3, p.353-358, 1995.

SILVA, M.T.B. da. Controle da lagarta da soja (Anticarsia gemmatalis Hübner, 1818 - Lepidoptera: Noctuidae). II. Baculovirus anticarsia. Ciência Rural, Santa Maria, v.22, n.3, p.261-265, 1992 .

SILVA, M.T.B. da. Controle da lagarta da soja (Anticarsia gemmatalis Hübner, 1818 - Lepidoptera: Noctuidae). IV. Controle biológico natural. Ciência Rural, Santa Maria, v.23, n.2, p.127-132, 1993

SILVA, M.T.B. da. et al. Avaliação de inseticidas sobre predadores das pragas da soja, em dez anos agrícolas, no Brasil. Trigo e Soja, Porto Alegre, n.96, p.3-16, 1988 . 